\title{
Transatlantica
}

Revue d'études américaines. American Studies Journal

\section{Wallace Stevens : d'une version officieuse de la philosophie}

\section{Axel Nesme}

\section{(2) OpenEdition}

1 Journals

\section{Édition électronique}

URL : https://journals.openedition.org/transatlantica/6342

DOI : $10.4000 /$ transatlantica.6342

ISSN : $1765-2766$

Éditeur

Association française d'Etudes Américaines (AFEA)

Référence électronique

Axel Nesme, «Wallace Stevens : d'une version officieuse de la philosophie », Transatlantica [En ligne],

1 | 2013, mis en ligne le 26 février 2014, consulté le 01 février 2023. URL : http://

journals.openedition.org/transatlantica/6342 ; DOI : https://doi.org/10.4000/transatlantica.6342

Ce document a été généré automatiquement le 1 février 2023.

\section{(c) (i) (9)}

Creative Commons - Attribution - Pas d'Utilisation Commerciale - Pas de Modification 4.0 International - CC BY-NC-ND 4.0

https://creativecommons.org/licenses/by-nc-nd/4.0/ 


\title{
Wallace Stevens : d'une version officieuse de la philosophie
}

\author{
Axel Nesme
}

1 Le présent travail a pour point de départ la lecture de l'étude philosophique de Wallace Stevens que nous a livrée Simon Critchley, et dont la thèse est que l'œuvre du poète gravite autour de la «question centrale de l'épistémologie, celle du rapport entre la pensée et les choses, entre l'esprit et le monde » (Critchley, 2005, 22) ${ }^{1}$. Selon Critchley, la poésie de Stevens ne réduit pas davantage le réel à un " enchaînement de fictions ", comme le prétend Joseph Riddel, qu'elle n'assimile le monde à une pure représentation comme le suggère $\mathrm{H}$. Bloom (Critchley, 27). Transfiguration et contraction constitueraient les deux modalités de présentation du réel que Stevens explore tour à tour dans une poésie qui témoignerait d'une appréhension phénoménologique du monde en tant que lieu « dans lequel nous sommes entièrement immergés et dont nous ne nous distinguons pas fondamentalement » (Critchley, 29). La poésie stevensienne se donnerait « pour tâche de nous donner le sentiment du monde tel qu'il est » (Critchley, 30), ce qui la placerait dans le prolongement de l'idée heideggérienne bien connue selon laquelle "l'homme habite en poète", selon la formule que le philosophe emprunte à Hölderlin pour en faire le titre d'un texte réuni dans ses Essais et conférences.

2 Pourtant, malgré cette invocation de la figure tutélaire de Heidegger, le texte de Critchley n'approfondit guère les éventuelles affinités qui lient celui-ci à la poésie de Stevens. Pour l'essentiel, c'est une version post-kantienne de Stevens qu'il nous livre, ainsi lorsqu'il écrit que " pour Stevens comme pour Kant, la réalité n'est réellement réelle que pour un auditoire réel composé d'individus réels, mais elle est tout entière traversée par un contenu conceptuel dont la source ultime est l'imagination » (Critchley, 37), ou encore, lorsqu'il assimile l'œuvre de Stevens à « une version poétisée de l'idéalisme transcendantal » (Critchley, 53).

3 La vérité du poème en tant que "version officieuse de l'être " (Stevens, 1997, 667) 2 consisterait dès lors dans le parfait ajointement de l'imagination à la réalité qu'il fait éprouver émotionnellement au lecteur. En effet il existe entre l'esprit et le monde 
« une sorte d'harmonie» (Critchley, 39) qui n'exclut pas que celui-ci soit, par l'entremise de l'imagination, soumis à divers « changement[s] d'aspect » (Critchley, 40). On n'est guère surpris, dès lors, de voir Critchley reconduire à propos de Stevens une conception rimbaldienne de la poésie comme alchimie du verbe et pouvoir de transmutation-transfiguration du réel à laquelle Stevens souscrit d'ailleurs lui-même, puisqu'il définit l'imagination comme faculté qui confère une "noblesse $»^{3}$ au réel (Stevens, 643-65).

Dans un deuxième temps de son étude, toutefois, Critchley observe dans la poétique de Stevens un renversement au détriment de la conception quelque peu ornementale qui prévalait jusque là, et une mise en avant d'un Stevens plus nouménal, si l'on peut oser la formule, c'est-à-dire d'un Stevens de la chose en soi « sans les atours de l'incantation poétique et le clinquant de l'ornementation » (Critchley, 63), confronté au mutisme et à l'étrangeté des choses ramenées à leur simple présence, que les poèmes tardifs constatent simplement, cessant d'opposer à la pression de la réalité la pression opposée de l'imagination.

5 Je souhaiterais, dans les pages qui vont suivre, commencer par montrer les limites de la perspective "épistémologique » revendiquée par Critchley à propos de Stevens, puis m'intéresser d'un peu plus près que ne le fait le critique à la pertinence du questionnement heideggérien et post-heideggérien par rapport à quelques textes de Stevens qui se prêtent à une mise en résonnance dont je n'ignore pas, tout en espérant m'y soustraire, qu'elle s'expose aux objections qu'Adorno, dans « Parataxe » (Adorno, 1984, 307-50), souleva à propos de la lecture heideggérienne de Hölderlin 4 . Aussi bien ne s'agira-t-il ici que d'analyser des convergences locales, des moments d'intersection non moins « officieux » et provisoires que ne l'est la version de l'être que nous propose Wallace Stevens. J'examinerai tout d'abord le contexte d'émergence de l'œuvre stevensienne "en temps de nécessité », en liaison avec la fonction originante de cette œuvre, en tant que celle-ci trouve son point focal dans la deixis, événement de langage renvoyant à la dimension négative de la voix comme fondement de la parole. Ceci m'amènera à m'interroger sur le rôle de la syllabe chez Stevens et m'intéresser à certains textes dont l'événement central est le retrait de l'étant permettant une approche de la poésie comme expérience ${ }^{5}$.

\section{Limites de la perspective épistémologique}

Qu'en est-il, pour commencer, du questionnement "épistémologique » auquel se ramène l'œuvre de Stevens selon la thèse évoquée en introduction? Un bref examen d'un poème qui semble procéder d'un tel questionnement en montre aussi d'emblée les limites.

The Glass of Water

That the glass would melt in heat,

That the water would freeze in cold,

Shows that this object is merely a state,

One of many, between two poles. So,

In the metaphysical, there are these poles.

Here in the centre stands the glass. Light

Is the lion that comes down to drink. There

And in that state, the glass is a pool.

Ruddy are his eyes and ruddy are his claws

When light comes down to wet his frothy jaws 
And in the water winding weeds move round.

And there and in another state-the refractions,

The metaphysica, the plastic parts of poems

Crash in the mind-But, fat Jocundus, worrying

About what stands here in the centre, not the glass,

But in the centre of our lives, this time, this day,

It is a state, this spring among the politicians

Playing cards. In a village of the indigenes,

One would have still to discover. Among the dogs and dung,

One would continue to contend with one's ideas. (Stevens, 181)

7 Ce texte débute sur une analogie qui se paraphrase sans difficulté. Un verre d'eau est un état temporaire entre deux extrêmes. Il suffit que la température baisse pour que l'eau qu'il contient gèle, ou au contraire qu'elle augmente pour que le contenant luimême fonde. De même dans le domaine «métaphysique » des idées, on n'a affaire qu'à des différences d'états contenues entre des extrêmes. La lumière qui, ici, dans le monde empirique, se reflète dans l'eau du verre devient là, dans le monde des idées poétiques, un lion qui s'abreuve dans une flaque d'eau au fond de laquelle des algues s'agitent. Que les conditions varient, et les algues acquièrent un degré supplémentaire d'abstraction : elles ne forment plus maintenant qu'un chaos d'éléments idéels dits "metaphysica", soit la matière malléable dont est fait le poème, qui s'entrechoquent dans l'imagination créatrice. On a donc en réalité deux métaphores dont une seulement fait l'objet d'une médiation discursive par l'entremise de la copule : «light/ Is the lion ». La deuxième, simplement sous-entendue relie "pool " à "mind». Or, c'est précisément là où la construction analogique, reposant sur des équivalences terme à terme, se met à boiter, que commence le poème dont il est d'ailleurs dit très explicitement que les composantes sont à ce point "malléables " qu'elles menacent d'effondrement toute élaboration conceptuelle, à commencer par celle que les deux strophes initiales du poème ont entrepris de tisser.

A la faveur d'un nouveau changement d'état, la position centrale occupée par le verre devient la métaphore de toute préoccupation au cour de l'existence de l'énonciateur et de son interlocuteur, tandis que ce qui était précédemment une modalité créatrice d'appréhension du réel défini par ses coordonnées dans l'espace se mue à présent en vague inquiétude quant au temps présent («this time, this day, [...] this spring among politicians »).

Le poème procède-t-il d'un questionnement épistémologique quant aux modalités selon lesquelles un même objet se diffracte dans la subjectivité qui l'appréhende, ou porte-t-il sur les avatars de la métaphore en fonction des divers degrés de mise en présence ou d'effacement des termes qui la composent? Ou bien se contente-t-il de parodier les faciles attraits de la pensée binaire et son enjeu est-il extérieur aux considérations qui précèdent? Cette hypothèse mérite d'être envisagée si l'on prend en compte deux données complémentaires. Car en fait de binarisme, on remarque en premier lieu que le couplet rimé qui clôt la deuxième strophe se signale surtout par sa symétrie quelque peu factice, surtout dans un poème où la rime est notoirement absente et dont les rythmes sont moins prévisibles que les iambes qui se succèdent machinalement dans ces deux vers : « Ruddy are his eyes and ruddy are his claws/ When light comes down to wet his frothy jaws. » En deuxième lieu, ce qui attire l'attention du lecteur autant que l'argument du poème est sans doute l'insolite apostrophe qui vient rompre l'énumération monotone des diverses réfractions «métaphysiques » du verre d'eau éponyme. Fait singulier, les vers qui thématisent le chaos des ingrédients du poème 
sont aussi ceux où se produit sa plus retentissante catastrophe verbale au moment où la méditation se mue en adresse lancée à ce "fat Jocundus ", personnage dont on ignore s'il est le double jovial du poète ou le représentant du lecteur. La juxtaposition d'un adjectif monosyllabique d'origine anglo-saxonne avec un substantif d'origine latine, l'appariement incongru du nom masculinisé de la Joconde avec un épithète figurant rarement parmi les connotations de la Mona Lisa, et moins encore dans l'arsenal lexical de la spéculation métaphysique; enfin, l'irruption de l'apostrophe après une longue série de déclaratives: autant de données qui infléchissent le tour argumentatif du poème, qui imposent à la visée illustrative d'une thèse épistémologique qu'on pourrait lui prêter plus qu'un simple détour ou qu'un simple dé-centrement. Elles y opèrent un changement d'état simultané à l'adoption d'un idiome où latin de cuisine et vocabulaire philosophique, en se mélangeant, permettent à Stevens de jeter les prolégomènes d'une plastique métaphysique.

En cela, Stevens correspond bien à la figure quasi oxymorique du "Connoisseur of Chaos » (Stevens, 194) qui consacre le poème de ce titre à la multiplicité grouillante des faits («the squirming facts») et aux précaires relations qui se nouent entre eux. S'interrogeant sur la possibilité qu'à partir d'un chaos de vérités partielles émerge une vérité statique (ou "Plantagenet » [Stevens, 195] comme Stevens la nomme par allusion à l'étymologie de l'ancienne dynastie anglaise ${ }^{6}$ ), le poète opère un détour par la pensée emersonienne. En effet, dans la figure de l'homme dit "pensif» dont Stevens écrit: "He sees that eagle float/ For which the intricate Alps are a single nest ", il y a sans doute une allusion à l'idée emersonienne selon laquelle toute cohérence des actions humaines est assimilable à un effet de perspective :

There will be an agreement in whatever variety of actions, so they be each honest and natural in their hour. For of one will, the actions will be harmonious, however unlike they seem. These varieties are lost sight of at a little distance, at a little height of thought. One tendency unites them all. The voyage of the best ship is a zigzag line of a hundred tacks. See the line from a sufficient distance, and it straightens itself to the average tendency. (Emerson, 266)

11 Pour autant, il s'en faut de beaucoup que ce «connaisseur » nous présente le modèle d'une subjectivité dans laquelle s'opère la synthèse du chaos. Car c'est de loin qu'il observe le vol de l'aigle pour qui les Alpes se ramènent à l'unité d'un seul nid, et rien n'indique que sa perspective se confonde avec celle de l'oiseau : au contraire l'absence de rime dans l'ultime couplet du poème marque l'irréconciliable divergence des regards, l'impossibilité de faire coïncider ordre et désordre.

\section{Une version de l'être : la poésie stevensienne en temps de nécessité}

12 C'est pourquoi on n'ira pas chercher du côté de l'épistémologie le dialogue le plus fécond que Stevens noue avec la philosophie, mais plutôt dans le voisinage d'une pensée qui s'est pour une part élaborée à la lecture de la poésie tout comme la poésie de Stevens n'a elle-même cessé de s'interroger sur ce qu'un de ses poèmes nomme «The Pure Good of Theory » (Stevens, 289).

13 Commençons donc par examiner un poème au titre prometteur : "The Role of the Idea in Poetry "(Stevens, 457). La parole philosophique dont ce texte se fait l'écho est l'effet d'une hantise : suggérée au philosophe par le spectre de son père, semblable aux nuées 
dont l'amoncellement donne un contour visible au tardif («clouds that hang lateness on the sea») elle retentit au soir, dans un "temps après le temps" nettement postérieur à la floraison des mythes, mais qui n'exclut pas qu'y naisse une forme évocatrice de la statuaire antique et susceptible de conférer une dignité à ce temps de dénuement :

Therein, day settles and thickens round a form -

Blue-bold on its pedestal - that seems to say,

"I am the greatness of the new-found night." (Stevens, 457)

14 Tel est le rôle dévolu à l'imagination que Stevens nomme " the necessary angel of the earth » (Stevens, 423), formule qu'il ne faudrait sans doute pas traduire littéralement par "nécessaire ange de la terre", mais plutôt par "ange de la terre au temps de nécessité ». Ce temps de nécessité est la modernité comme moment de retrait du divin dans lequel Stevens voit l'occasion de célébrer les noces du monde et de l'imagination comme il le fait dans «The Sense of the Sleight-of-Hand Man » (Stevens, 205), où la maison déserte qu'envahissent des flots de lumière blanche, la roue de feu qui auréole le soleil par simple réfraction de ses rayons, ou encore la colombe "à l'œil de grenadine », ne font pas signe vers le numineux ou la symbolique biblique mais, pour celui qui ignore cette dimension, campent le décor d'une existence ramenée aux cinq sens et qui prospère au cœur de l'hiver de la croyance :

[...] The wheel survives the myths.

The fire eye in the clouds survives the gods.

To think of a dove with an eye of grenadine

And pines that are cornets, so it occurs,

And a little island full of geese and stars:

It may be that the ignorant man, alone,

Has any chance to mate his life with life

That is the sensual, pearly spouse, the life

That is fluent in even the wintriest bronze. (Stevens, 205)

15 Si l'ange de Stevens est « nécessaire ", c'est donc au sens que revêt l'épithète " dürftig » dans le fragment de vers de Hölderlin qui sert de titre à l'avant-dernier chapitre de Chemins qui ne mènent nulle part : «Wozu Dichter in dürftiger Zeit ». Dans la traduction française de ce vers (« pourquoi des poètes en temps de détresse? » [Heidegger, 1962, 323]), l'on perd quelque peu de vue le sens premier du mot allemand qui signifie " nécessiteux », "frappé de nécessité ». Or, c'est bien pourtant parce que le temps des "Aurores de l'automne » que clôt le poème « Angel Surrounded by Paysans » (Stevens, 423) est un temps de nécessité ; c'est, a fortiori, en raison du même dénuement poussé à son comble dans le recueil suivant, The Rock (qui sera aussi le dernier dans l'œuvre de Stevens), que la nécessité du poème s'impose avec plus d'évidence. Françoise Dastur, dans sa glose de Heidegger, écrit à ce propos :

Voir et dire l'unité du divers et la divinité du tout, voilà en quoi consiste la tâche de l'existence poétique. Et ce n'est que lorsque le divin est séparé de la réalité et que le caractère poétique de celle-ci n'est plus perçu ni expérimenté et qu'elle ne se présente plus que comme facticité nue, qu'il est alors plus que jamais besoin de poètes et de poésie. C'est pourquoi à la fin de la septième strophe de son élégie Brot und Wein, «Pain et vin », que Hölderlin compose au cours de l'hiver 1800 et qui dit le retrait du divin, on trouve la question adressée à son ami, le poète Heinse, auquel le poème est dédié : « Wozu Dichter in dürftiger Zeit? 》 (Dastur, 2005, 146)

16 Ainsi la forme indistincte qui, debout sur son piédestal, proclamait «I am the greatness of the new-found night » figurerait à bon droit parmi ces "poètes du temps de la détresse, de cette nuit du monde qui est pourtant aussi une nuit sacrée - car le retrait 
du divin est encore une manière pour le divin de régner - [qui] "apportent aux mortels la trace des dieux enfuis dans l'opacité de la nuit du monde" " (Dastur, 146).

C'est pourquoi la figure de l'ange, chez Stevens, ne témoigne d'aucune religiosité : elle est tout au plus une version moderne du daimon qui dépersonnalise à dessein l'origine même de l'œuvre afin d'éviter toute méprise quant à l'origine de celle-ci, notamment celle qui consisterait "à voir en l'artiste l'origine de l'œuvre, alors qu'il en est simplement la cause » (Dastur, 46). De fait, Stevens délègue, mythifie ou fictionnalise volontiers la fonction originante. On l'observe dans ses écrits en prose, le titre de l'essai "The Figure of the Youth As Virile Poet » (Stevens, 666-85) montrant bien que le poète ne se conçoit qu'à travers les masques qu'il revêt, mais aussi dans « The Idea of Order at Key West » (Stevens, 105) où le sujet poétique se dépeint en spectateur d'une figure féminine aux contours indécis, réduite au pronom personnel «she », qui par son chant ordonne l'océan et fait advenir un monde. "L'artiste sera compris comme [la] causa efficiens [de l'œuvre d'art], c'est-à-dire comme la «chose » qui vient originellement, avant le produit lui-même, à savoir l'Ur-sache, alors que l'origine, Ur-sprung, n'est pas une chose originelle, mais le fondement (Grund) duquel jaillit (springt) l'œuvre d'art et ce qui la rend possible et nécessaire" (Dastur, 47). Ce fondement est ce que Stevens nomme "the ever-hooded, tragic-gestured sea ", soit moins un horizon de sens qu'un espace d'où émane ce qui fait signe vers la nomination tout en demeurant voilé, maintenu à ce que Stevens nomme "theatrical distances", désignant par là non seulement une transfiguration du lointain, mais un lointain devenu théâtre, un ordonnancement de la distance en scène où un sens est susceptible de venir se jouer.

Afin de " rendre visible l'avènement de la vérité » (Heidegger, 1962, 43) tel que celui-ci se produit dans l'œuvre d'art, Heidegger propose dans "L'Origine de l'œuvre d'art » une œuvre non figurative (contrairement aux Sabots de Van Gogh évoqués au début de ce chapitre de Chemins qui ne mènent nulle part [Heidegger, 1962, 32 sq]) : le temple grec par la simple existence duquel «dans l'entaille de la vallée [...] le Dieu peut être présent » (Heidegger, 1962, 44). Par le périmètre que délimite son enceinte, «l'œuvretemple [...] dispose et ramène autour d'elle l'unité des voies et des rapports, dans lesquels naissance et mort, malheur et prospérité, victoire et défaite, endurance et ruine donnent à l'être humain la figure de sa destinée ». Ainsi le chant entendu à Key West, outre qu'il découpe l'étendue marine en « zones » et en « portails » à la manière dont "par sa sûre émergence " le temple rend "visible l'espace invisible de l'air " (Heidegger, 1962, 44), est porteur du sens de l'origine (« Words [...]/ of ourselves and of our origins ») (Stevens, 106) et du destin dont est lourd le geste "tragique » qui s'y esquisse avant même que ne retentisse le poème. Dans "The Man With the Blue Guitar " (Stevens, 135), de même, la mélodie de l'instrument circonscrit un espace (musical) et donne lieu aux choses: «The tune is space. The blue guitar// Becomes the place of things as they are,/ A composing of senses of the guitar » (Stevens, 137). «Les œuvres ont [...] la capacité de faire surgir originellement le monde » écrit à ce propos $\mathrm{F}$. Dastur dans son exégèse de Heidegger (Dastur, 51) : c'est le même pouvoir d'installation (Dastur, 55) d'un monde que Stevens prête à la femme dans «The Idea of Order at Key West » :

[...] Then we,

As we beheld her striding there alone,

Knew there never was world for her

Except the one she sang and, singing, made. (Stevens, 106) 


\section{rappelle Dastur :}

Ce qui est [...] pro-duit dans l'ouvert par l'intermédiaire de l'œuvre d'art, c'est la terre en tant qu'elle se renferme constamment en elle-même. Le verbe herstellen a aussi en allemand le sens de restaurer et de restituer quelque chose dans son état antérieur, de sorte qu'il est possible de comprendre la pro-duction de la terre comme un rétablissement de l'ouvert qui incline constamment à se refermer. [...] L'installation du monde et la production de la terre sont inséparables l'une de l'autre, tout comme l'éclaircie et l'occultation [...] L'œuvre d'art au sein de laquelle ces deux processus prennent place est le lieu d'un conflit entre monde et terre, ce qui veut dire que l'œuvre d'art est ce conflit lui-même, et non pas une simple représentation de celui-ci. (Dastur, 56)

De fait, sur le plan de la simple représentation, il est vrai que dans le texte de Stevens, éclaircie et occultation oscillent au gré des mouvements de la mer, de sorte que ce qui se manifeste apparaît rarement sans que soit ménagée l'hypothèse de son immédiat reflux. Ainsi, la voix de la femme, dans « The Idea of Order... » rend le ciel plus proche juste avant qu'il s'efface dans la nuit: «It was her voice that made/ The sky acutest at its vanishing ". Mais c'est aussi dans la langue même du poème que cette vacillation résonne puisque si les sonorités des mots qui disent la mer, au tout dernier vers, sont "plus vives", les démarcations qu'ils instaurent sont aussi "plus spectrales", maintenant le conflit qu'évoque la citation précédente dans la logique contradictoire de ces deux comparatifs, identiques morphologiquement, mais sémantiquement opposés.

21 Comment, cependant, réconcilier cette dimension pulsatile du réel avec un poème comme « The Man on the Dump » (Stevens, 185) où il semble s'agir au contraire, comme le souligne volontiers Critchley dans son portrait de Stevens en épistémologue, de restaurer avec le réel une idylle qui, si elle se déroule au clair de lune, n'en cherche pas moins à faire l'économie de la médiation de l'image romantique, afin de mieux saisir et figer le moment de pure apperception de l'objectivité, du « the » primitif ?

[...] and the moon comes up as the moon

(All its images are in the dump) and you see

As a man (not like an image of a man),

You see the moon rise in the empty sky.

[...] Did the nightingale torture the ear,

Pack the heart and scratch the mind? And does the ear

Solace itself in peevish birds? Is it peace,

Is it a philosopher's honeymoon, one finds

On the dump? Is it to sit among the mattresses of the dead,

Bottles, pots, shoes and grass and murmur aptest eve:

Is it to hear the blatter of grackles and say

Invisible priest; is it to eject, to pull

The day to pieces and cry stanza my stone?

Where was it one first heard of the truth? The the. (Stevens, 185)

La conclusion de cette réécriture stevensienne du «heap of broken images » sur les décombres duquel Eliot compose The Waste Land, est sans doute ce qui mérite le plus qu'on s'y attarde. En effet, dans la très grande pauvreté, le dénuement de son tout dernier vers, touchant à son paroxysme avec l'énigmatique et assez disgracieux «The the » qui lui sert de point d'orgue, il est tentant de lire, non pas quelque ultime effort pour saisir l'objet réduit à son épure, mais plutôt une instanciation de ce que Heidegger nomme le «tracé-ouvrant de la parole en son déploiement [...] où vient se dire, où se 
dédit ou bien s'interdit-se montre ou se dérobe- ce qui vient en présence et ce qui sort de la présence » (Heidegger, 1976, 240) tel que celui-ci se trace par le moyen de la deixis, soit ce que le philosophe nomme die Sage, « la dite »:

Suivant l'emploi le plus ancien du mot, nous entendons la Dite à partir du dire compris en tant que montrer, et nous employons pour nommer la Dite, dans la mesure où, en elle, repose la parole en son déploiement, un vieux mot, bien attesté, mais éteint: la monstre (die Zeige). Pronomen demonstrativum a été traduit par: Zeigewörtlin (l'index spirituel).

Ce qui se déploie dans la parole est la Dite en tant que monstre (Das Wesen der Sprache ist die Sage als die Zeige »). Qu'elle montre ne se fonde pas en Dieu sait quels signes, mais au contraire: tous les signes tirent origine d'un montrer dans l'horizon et pour intentions duquel seulement ils peuvent être signes. (Heidegger, 1976, 240-1)

\section{Deixis, voix et syllabe}

«La deixis, l'indication; ne montre pas simplement un objet innommé, mais avant tout l'instance même du discours, son avoir lieu ", écrit Agamben (Agamben, 1997, 57), qui montre avec finesse que derrière la «transcendance de l'être par rapport à l'étant, du monde par rapport à la chose " se dissimule la «transcendance de l'événement de langage par rapport au discours ", soit ce qui relève de la voix : "C'est uniquement parce que le langage permet, à travers les shifters, de faire référence à sa propre instance, que quelque chose comme l'être et le monde, s'ouvrent à la pensée. » C'est pourquoi « la transcendance de l'être et du monde [...] que Heidegger identifie comme la structure fondamentale de l'être au monde " passe tout entière par le jeu des déictiques et de la voix qui les sous-tend:

L'énonciation et l'instance de discours ne peuvent être identifiées comme telles qu'à travers la voix qui les profere, et [...] ce n'est qu'en lui supposant une voix que quelque chose comme un avoir-lieu du discours peut être montré [...]

La voix, ainsi considérée, se révèle dès lors pure intention de signifier, pur vouloirdire, où quelque chose se donne à comprendre sans qu'il ne se produise encore aucun événement de signification déterminé. (Agamben, 70)

Or, dans la démonstration d'Agamben qui se propose comme un au-delà de la déconstruction derridienne de la phonè, la voix que supposent les shifters ne se réduit pas au « flux sonore émis par l'apparat phonatoire, de même que le Je, le locuteur n'est pas simplement l'individu psychosomatique d'où provient le son. » En effet, « une voix comme simple son [...] ne peut aucunement renvoyer à l'instance du discours en tant que tel, ni ouvrir la sphère de l'énonciation ", même si elle renvoie comme un indice à la présence dont elle émane :

La voix, la phonè animale, est, certes, présupposée par les shifters, mais comme ce qui doit nécessairement être supprimé afin que le discours signifiant ait lieu. L'avoir-lieu du langage entre la suppression de la voix et l'avènement de signification est l'autre Voix; qui, dans la tradition métaphysique, constitue l'articulation originelle [...] du langage humain. Mais, en tant que cette Voix [...] a le statut d'un non-plus (voix) et d'un non-encore (signifié) elle constitue nécessairement une dimension négative. Elle est le fondement, mais au sens où elle est ce qui s'abîme et disparaît afin que l'être et le langage aient lieu ». (Agamben, 74-75)

Agamben ajoute que "la dimension négative, qui constitue le seul shifter possible" entre langue et parole et dont le lieu est la Voix, « est présente également dans la linguistique moderne à travers le concept de phonème, de cet étant purement négatif et insignifiant, qui est, toutefois, précisément ce qui ouvre et rend possible la 
signification et le discours » (Agamben, 151). Or, à propos du dernier vers de «The Man on the Dump ", on peut certes pointer qu'il suffit d'une répétition pour que le même signifiant soit différemment accentué et prononcé, qu'un espacement le traverse du fait de son redoublement. Le retour rêvé à une épure d'énoncé poétique, un énoncé précédant la nomination, le «das " avant même qu'il y ait « das Wort ", dirait-on en songeant au commentaire heideggérien de Stefan George, fait jaillir au cœur de la deixis sa propre refente, le deuil du référent simultané à l'auto-désignation du déterminant. De sorte que si dans son discours, le dernier vers entérine l'existence de la vérité, dans sa facture il signale une faille au cœur de la monstration.

Mais il est également intéressant de noter que l'article redoublé, en tant qu'il se prête à deux réalisations différentes, est singularisé comme " "son de la langue" (c'est-à-dire de ce qui, par définition, ne peut avoir de son)», de sorte qu'il "apparaît singulièrement proche de l'idée heideggérienne d'une "Voix silencieuse" et d'un "son du silence" ", faisant ainsi ressortir «le rapport essentiel entre le langage et la mort [qui] a - pour la métaphysique - son lieu dans la Voix » (Agamben, 152). Ainsi Voix de l'être et phonème renvoient toutes deux au « fondement négatif originel de la parole humaine. Faire l'expérience de la Voix [...] signifie être capable d'une autre mort, qui ne soit plus simplement le trépas et constitue la possibilité la plus propre et la plus insurmontable de l'existence humaine, sa liberté » (Agamben, 152).

De ce point de vue, le déictique est le signifiant de l'événement par excellence en tant qu'accès de l'homme à la mort qui lui revient en propre, si l'on entend bien le mot "événement» au sens que Heidegger met en lumière dans l'étymologie de son équivalent allemand, Ereignis, soit l'appropriement en tant que «statut» (Gesetz), loi qui « approprie les mortels par cela qu'elle les remet en propre à ce qui [...] se dit en s'adressant à l'homme dans la Dite " pour autant que celle-ci « délivre la manière d'être humaine en son propre » (Heidegger, 1976, 248-49).

C'est pourquoi les motifs qui, dans l'idiome stevensien, semblent les plus proches du phonème, celui de la syllabe et du cri, ne cessent (parfois conjointement) de refaire surface dans les textes de la vieillesse du poète (à partir de Transport to Summer), comme si par leur entremise, Stevens s'efforçait de saisir le degré élémentaire d'articulation signifiante qui serait l'affirmation d'une liberté au seuil de la disparition.

Dans « No Possum, No Sop, No Taters » (Stevens, 261) les substantifs mis en présence dans le titre entretiennent trop peu d'affinités sémantiques les uns avec les autres, tout comme avec le reste du poème qu'ils introduisent, pour qu'il reste de ce titre autre chose que le simple geste de la triple négation en un texte auquel Stevens eût fort bien pu donner le titre "Description without place» (Stevens, 296), puisque son enjeu consiste à produire un non-lieu descriptif. De fait, la négation de l'étant constitue le fil directeur de ce poème où la nature - nature morte si l'on prend la formule au pied de la lettre - est placée sous le signe de la privation, comme si ce qui cherche à s'y déployer supposait que le poète renonce «à avoir en sa puissance le mot en tant que nom qui exhibe un étant fixé » (Heidegger, 1976, 213) mais cherchait plutôt à en dire la condition d'émergence en n'évoquant les éléments du paysage que par ce qui leur fait défaut, qu'il s'agisse du soleil qui l'a déserté (" He is not here, the old sun,/ As absent as if we were asleep ») ou des tiges amputées et décapitées :

In this bleak air the broken stalks

Have arms without hands. They have trunks

Without legs or, for that, without heads. (Stevens, 261) 
«Le mot ne donne pas le fondement de la chose », écrit Heidegger, « le mot laisse venir en présence la chose comme chose » (Heidegger, 1976, 218). La condition, au contraire, « est, pour quelque chose d'étant, ce qui est son fondement [...] La condition donne le fondement et fonde ». C'est seulement à partir de ce fondement qu'une expérience de la Voix devient possible, au sens où Agamben précisait la portée de ce terme, telle que celle-ci se déploie sous le double avatar du cri, puis de la syllabe-phonème :

They have heads in which a captive cry

Is merely the moving of a tongue.

[...]

It is in this solitude, a syllable,

Out of these gawky flitterings,

Intones its single emptiness,

The savagest hollow of winter-sound. (Stevens, 262)

31 "Fondement négatif originel de la parole humaine ", selon la formule d'Agamben déjà citée, la syllabe l'est bien en effet ici en ce sens qu'elle ne fait rien entendre sinon deux déclinaisons successives du néant comme vide sonore qu'elle creuse. Quant au geste critique qui met en résonnance ces données négatives, il est lui-même partie prenante de cet avènement ex nihilo de la vérité qui, dans l'œuvre, conjoint solidairement création et « sauvegarde» :

Dans l'œuvre, c'est l'avènement de la vérité, qui est à l'œuvre et précisément selon le mode de l'œuvre. C'est pourquoi nous avions déterminé d'avance l'essence de l'art comme la mise en œuvre de la vérité. Or cette détermination est sciemment ambiguë. D'une part elle dit : l'art, c'est la constitution, en une stature, de la vérité qui s'institue : ce qui advient par la création comme production de la déclosion de l'étant. Mais, en même temps, mettre en œuvre signifie : mettre en marche et faire parvenir à l'avènement l'être-œuvre : ce qui advient par la sauvegarde. L'art est alors : la sauvegarde créant la vérité dans l'œuvre. L'art est donc un devenir et advenir de la vérité. Et la vérité ? Provient-elle du Rien? Assurément, si par Rien on entend la pure et simple négation de l'étant, celui-ci étant représenté comme ce donné habituel et disponible, qui précisément, par la seule instance de l'œuvre, s'ébranlera et s'avèrera être l'étant qui n'était vrai que putativement. (Heidegger, 1962, 80-1)

Une telle négation de l'étant ne se résume pas à un moment tardif dans l'œuvre de Stevens. En effet, dès Harmonium "The Snow Man» (Stevens, 8) est aussi this no man auquel se résume la figure du poète dans le texte qu'on vient d'étudier. Plus que le long et baroque "The Comedian as the Letter $C$ », ce poème pourrait à bon droit passer pour le moment inaugural de l'œuvre de Stevens, notamment en raison de son extrême dépouillement, terme qu'il ne faut pas entendre ici comme dénotant un état, mais un procès de mise à l'écart préfigurant ce que Stevens, dans " The Rock », nommera " cure of the ground» (Stevens, 446), et visant non à restituer un «donné habituel et disponible ", mais au contraire à garantir les conditions d'une indisponibilité préalable à toute instauration :

One must have a mind of winter

To regard the frost and the boughs

of the pine-trees crusted with snow;

And have been cold a long time

To behold the junipers shagged with ice,

The spruces rough in the distant glitter

of the January sun; and not to think

of any misery in the sound of the wind,

In the sound of a few leaves, 
Which is the sound of the land

Full of the same wind

That is blowing in the same bare place

For the listener, who listens in the snow,

And, nothing himself, beholds

Nothing that is not there and the nothing that is. (Stevens, 8)

\section{(Heidegger, 1968, 58) : \\ March ... Someone has walked across the snow, \\ Someone looking for he knows not what. \\ It is like a boat that has pulled away \\ From a shore at night and disappeared. \\ It is like a guitar left on a table \\ By a woman, who has forgotten it. \\ It is like the feeling of a man \\ Come back to see a certain house. \\ The four winds blow through the rustic arbor, \\ Under its mattresses of vines. (Stevens, 434-5)}

surgissement du poétique passe ici par une série de négations au premier rang desquelles figure celle du pathos volontiers associé aux descriptions hivernales. Projeter l'expression d'une douleur dans le hurlement du vent serait en effet un sûr moyen de ne pas laisser l'œuvre être œuvre en l'assimilant à un « objet susceptible de provoquer en nous Dieu sait quels "états d'âme” " (Heidegger, 1962, 78).

4 Les jalons ainsi posés dans un des tout premiers textes de Stevens se retrouvent, à l'autre extrémité de la carrière du poète, dans « Vacancy in the Park » (Stevens, 434), texte qui partage avec « The Snow Man » non seulement son thème hivernal, mais aussi sa façon de mettre en scène ce que Heidegger nomme «recul de l'existant»

Les points de suspension qui interrompent d'entrée de jeu la progression du poème semblent ici ne faire qu'un avec les traces de celui qui a marché dans la neige. Le vestige d'une présence et la marque d'un non-dit qui ponctue le blanc de la page font plus que se superposer : ils se confondent. Est-ce le vide pris en son sens absolu que désigne le titre du poème, ou une place laissée vacante, mais faite pour être remplie, comme dans un hôtel où il reste des chambres disponibles? Les points de suspension, de fait, signalent la possibilité que quelque chose vienne occuper cet espace, par exemple une série de comparaisons, dont le nombre sera exactement égal au nombre de points qui trouent le premier vers. Mais chacune de ces comparaisons n'aura d'autre effet que d'augmenter l'indétermination initiale. Ce vide est semblable à un bateau absent dont il ne reste pas même le sillage, qui a levé l'ancre (négation du lieu) pour s'éloigner la nuit (négation de la lumière et du jour) et disparaître (négation de l'apparaître). Il ressemble à une guitare oubliée, indice d'une présence qui n'est plus, et qui plus est, de la présence d'une femme qui, en oubliant l'instrument, a eu une absence : bref d'une absente souffrant d'absence qui est l'image inversée du promeneur lequel, s'il n'oublie pas ce qui lui appartient, ignore en revanche ce qu'il cherche. Ce vide est enfin analogue au néant référentiel des vers : «It is like the feeling of a man/ Come back to see a certain house ", puisque le lecteur, ignorant tout de cette maison, ne sait rien non plus du sentiment qu'elle peut éveiller.

Cet évidement du sens culmine avec l'introduction de la métaphore qui supplante les comparaisons précédentes. Les matelas de treilles qui forment le pavillon de verdure sont en effet une métaphore doublement paradoxale. D'abord parce qu'un matelas est rembourré à l'inverse d'un treillage qui se tresse autour du vide. D'autre part parce que 
la présence de la métaphore ne s'avère que si elle est prise comme métaphore in absentia : si « mattresses of vines » est le signifiant métaphorique du vide du titre.

Dans la logique du signifié, de la représentation, "mattresses of vines » relève du paradoxe. Sous l'angle du signifiant, le terme est au contraire bienvenu, puisqu'il soude les synonymes « matt » et «tress » qui redoublent le motif du tressage autour du vide. La métaphore advient alors à la faveur d'un retrait du signifiant «mattress» qui s'efface derrière la juxtaposition matt-tress, qui s'absente pour qu'en son sein l'absence se redouble en tant que ce vide autour duquel les fils d'un tressage forment leur écheveau au même titre qu'il est ce de part et d'autre de quoi la trame et la chaîne d'une natte se croisent.

L'indétermination croissante qui se propage ainsi au fil du texte génère un sentiment qui n'est peut-être pas étranger à celui du visiteur «de retour dans une certaine maison ». Ce sentiment dont le texte, sans le nommer, suggère qu'il naît face à "l'impossibilité essentielle de recevoir une détermination quelconque " (Heidegger, 1968, 58), évoque ce qui est dit de l'angoisse dans les pages bien connues de «Qu'est-ce que la métaphysique », où Heidegger écrit :

Toutes les choses et nous-mêmes, nous nous abîmons dans une sorte d'indifférence. Cela pourtant non point au sens d'une disparition pure et simple, mais dans leur recul comme tel, les choses se tournent vers nous [...] Il ne reste rien comme appui. Dans le glissement de l'existant, il ne reste et il ne nous survient que ce « rien». (Heidegger, 1968, 58)

Quelle est la nature de cette expérience dont le poème garde la trace ? Celle-ci, écrit Jean-Luc Lacoue-Labarthe, ne relève pas de l'anecdote: elle relève de ce que l'étymologie latine du mot, ex-periri, met en lumière. Elle est «la traversée d'un danger » (Lacoue-Labarthe, 1997, 30) du même ordre que ce qui affleure dans l'angoisse. "Si l'on parle d' "émotion poétique", ajoute Lacoue-Labarthe, il faut la comprendre comme émoi, ce qui veut dire : absence ou privation de moyens » (Lacoue-Labarthe, 33). Cette privation de moyens trouve sa paradoxale traduction dans le « vouloir-ne-riendire » du poème dont Lacoue-Labarthe précise qu'il «n'est pas un vouloir ne rien dire. Un poème veut dire, il n'est même que cela, pur vouloir-dire. Mais pur vouloir-dire le rien, le néant, ce contre quoi et par quoi il y a la présence, ce qui est. » (LacoueLabarthe, 33-34).

\section{La poésie de Wallace Stevens comme expérience}

Je voudrais, pour conclure cette étude, m'intéresser à un poème de Stevens qui nous propose, et ce n'est pas fortuit, le court récit d'une traversée qui est aussi celui d'une expérience au sens où on vient d'entendre ce terme, expérience dont le point d'aboutissement est un au-delà du figural dans l'avènement du pur vouloir-dire. A l'origine de ce diptyque hanté par le démon de l'analogie qu'est «Prologues to What Is Possible» (Stevens, 437), figure une comparaison que la première des deux sections s'attache à déployer : « There was an ease of mind that was like being alone in a boat at sea ». La tranquillité d'esprit du penseur méditant sur l'horizon des possibles se mue en barque dont les rameurs l'acheminent vers une destination qui se précise peu à peu. L'embarcation qui contient le voyageur est un pur produit de l'imagination: taillée dans la pierre, elle n'a gardé que l'éclat de celle-ci sans en avoir la densité qui ferait couler la barque. Les prologues du possible s'articulent donc sur fond d'impossible, et 
l'on devine sans mal que la traversée en question appelle un déchiffrement métaphorique. Plus exactement, et quoi qu'en dise Stevens lui-même dans son journal lorsqu'il écrit : « There is no such thing as metaphor of a metaphor » (Stevens, 921), elle demande à être lue en deux temps, comme métaphore simple, puis comme métaphore de la métaphore. En un premier temps, en effet, la barque improbable, composée de matière minérale en écho au titre du recueil, The Rock, ne transporte pas vers un audelà du familier. Autrement dit, les termes de la métaphore sont empruntés au monde des objets quotidiens, auxquels la figure se contente d'ajouter un « éclat » particulier, puisqu'aussi bien, la proue de la barque arbore un "speculum de feu» dont le flamboiement transfigure ce qui s'y reflète, et ses flancs, "tel le verre ", revêtent les couleurs de la mer. Quant au passager, il figure ici le destinataire du trope dont l'expérience herméneutique est assimilée à une traversée. Cette première figure présente les limites qui sont celles de toute comparaison ontique, dont Lacoue-Labarthe pointe qu'elle reste muette quant à la question de l'être :

S'agissant de la poésie, la représentation s'organise à partir de ce qu'on pourrait appeler la comparaison ontique (comparaison du déjà-présent avec le déjà-présent) d'où prennent naissance les figures ou les images: "les métaphores et autres tropes ", l'ensemble des tours qui permettent de définir un certain usage du langage comme "poétique ». Mesurée à l'exigence du questionner vers l'être ou la présence, la comparaison ontique, et par conséquent le "poétique ", relèvent de ce que Heidegger a dénoncé comme «idolâtrie » ou problématisé comme " pensée par modèles ». (Lacoue-Labarthe, 99-100)

41 Sur cette première analogie s'en greffe cependant une seconde. En effet, tout lecteur familier de la dualité vehicle/tenor, volontiers utilisée en anglais pour désigner le signifiant et le signifié de la métaphore, est prédisposé à appréhender la figure en termes de rapport entre un contenant et un contenu, et donc à lire à cette aune le lien qui unit non plus la barque à l'élément qui la porte, mais le passager à son embarcation. Cette lecture est d'autant plus prévisible que toute métaphore opérant par transfert de sens du propre vers le figuré (sens sur lequel jouait déjà le titre du recueil Transport to Summer), il semble particulièrement approprié d'en représenter le processus sous la forme d'un itinéraire vers un sens nouveau qui serait le produit de l'opération métaphorique, avec le passage par le non-sens que celle-ci suppose, tel que celui-ci est désigné par la « syllabe dénuée de sens » qui, dans la première section du poème, attire le voyageur vers la signification à venir.

On a ici un singulier télescopage des figures de la ressemblance, puisque le comparant de l'analogie initiale se mue à son tour en comparé de la métaphore comme acheminement vers un nouveau rapport comparant-comparé associant «syllable without meaning» et "the meaning into which he wanted to enter». En effet, le passager est à l'embarcation ce que le signifié (" meaning ») de la métaphore est à son signifiant («syllable»). Mais au terme de ce voyage métaphorique, c'est-à-dire de ce voyage comme métaphore de la métaphore, la métaphore est elle-même destinée à voler en éclat en même temps que la vie prend fin sur ce rivage loin de toute terre habitée :

As he traveled alone, like a man lured on by a syllable without any meaning,

A syllable of which he felt, with an appointed sureness,

That it contained the meaning into which he wanted to enter,

A meaning which, as he entered it, would shatter the boat and leave the oarsmen

quiet

As at a point of central arrival, an instant moment, much or little, 
Removed from any shore, from any man or woman, and needing none (Stevens, 438) débouche paradoxalement sur son retrait, la première section du poème de Stevens semble s'inscrire dans le paradigme du discours métaphysique au sein duquel Derrida montre que tout retrait n'est jamais que le redoublement d'un trait :

Le discours dit métaphysique ne peut être débordé, en tant qu'il correspond à un retrait de l'être, que selon un retrait de la métaphore en tant que concept métaphysique, selon un retrait du métaphysique, un retrait du retrait de l'être. Mais comme ce retrait du métaphorique ne laisse pas la place libre à un discours du propre ou du littéral, il aura à la fois le sens du re-pli, de ce qui se retire comme une vague sur le littoral, et d'un re-tour, de la répétition surchargeant d'un trait supplémentaire, d'une métaphore de plus, d'un re-trait de métaphore, un discours dont la bordure rhétorique n'est plus déterminable selon une ligne simple et indivisible, selon un trait linéaire et indécomposable.. Ce trait a la multiplicité interne, la structure pliée-repliée d'un re-trait. Le retrait de la métaphore donne lieu à une généralisation abyssale du métaphorique [...] (Derrida, 1987-1998, 80-1)

Il me semble néanmoins que cette syllabe qui a le «statut d'un non-plus (voix) et d'un non-encore (signifié) » relève bien davantage de ce qu'Agamben dit de la voix comme " pure intention de signifier, pur vouloir-dire, où quelque chose se donne à comprendre sans qu'il ne se produise encore aucun événement de signification déterminé " (Agamben, 70). Y accéder, c'est, selon la formule d'Agamben déjà citée, "faire l'expérience de la Voix [qui] signifie [...] être capable d'une autre mort, qui ne soit plus simplement le trépas et constitue la possibilité la plus propre et la plus insurmontable de l'existence humaine, sa liberté » (Agamben, 152).

L'expérience de cette syllabe comporte sa part de péril, car ce dont elle est le "prologue» (ainsi que l'annonce le titre) est l'entrée dans un au-delà de la ressemblance entre les étants, à commencer par celle qui unit un sujet aux «this and that " dans lesquels il se projette imaginairement et se reconnaît tant qu'il reste prisonnier d'une torpeur qu'il partage avec tous ceux dont les spéculaires spéculations restent tributaires de la pensée analogique :

The metaphor stirred his fear. The object with which he was compared Was beyond his recognizing. By this he knew that likeness of him extended Only a little way, and not beyond, unless between himself

And things beyond resemblance there was this and that intended to be recognized,

The this and that in the enclosures of hypotheses

On which men speculated in summer when they were half asleep. (Stevens, 438)

Observant l'interruption de la mimésis qui caractérise la poésie de Paul Celan, LacoueLabarthe écrit que chez Celan, «l'acte poétique consiste à percevoir, non à représenter. Représenter [...] ne peut se dire que du déjà-présent. Ce qui est "en train d'apparaître" ne se représente pas » (Lacoue-Labarthe, 99) :

[L]'acte poétique est extatique. L'exorbitant est la pure transcendance de l'étant [...] ouvert d'une certaine façon à l'u-topie, au lieu sans lieu de l'avènement. Ou dit autrement : l'acte poétique est catastrophique : rapport renversant à ce qui est et renversement, dans l'étant, vers le néant (l'abîme). (Lacoue-Labarthe, 99)

De fait, une sortie de soi attend le sujet dans l'angoisse qui se fait jour à la limite de la métaphore qui est aussi la limite où le sujet devient lui-même métaphore, porteur d'un sens dont le signifiant se refuse :

What self, for example, did he contain that had not yet been loosed,

Snarling in him for discovery as his attentions spread, 
As if all his hereditary lights were suddenly increased

By an access of color, a new and unobserved, slight dithering,

The smallest lamp, which added its puissant flick, to which he gave

A name and privilege over the ordinary of his commonplace-

A flick which added to what was real and its vocabulary,

The way some first thing coming into Northern trees

Adds to them the whole vocabulary of the South,

The way the earliest single light in the evening sky, in spring,

Creates a fresh universe out of nothingness by adding itself,

The way a look or a touch reveals its unexpected magnitudes. (Stevens, 438)

La menace sourde - ou la promesse - dont il est fait état dans ces vers tient à ce que le comparant est au-delà de toute ressemblance avec le comparé, au-delà même de toute intention de ressemblance, et se situe en-dehors de "l'enclos d'hypothèses " accessibles à la spéculation humaine. "Slight dithering", "puissant flick», ce supplément viendrait «ajouter au réel et à son vocabulaire» (439). Ce que Stevens appréhende ici n'est pas sans parenté avec cet « autre règne du mot » (Heidegger, 1976, 213) que Heidegger voit poindre dans l'expérience d'un échec de la nomination que relate le poème de George " Das Wort » :

Le poète doit abandonner son exigence et ne plus prétendre à ce que, en toute sûreté et à sa demande, le nom lui soit livré pour ce qu'il a établi et supposé véritablement étant. Cette supposition, et d'abord son exigence, voilà ce qu'il doit s'interdire. Le poète doit renoncer à avoir en sa puissance le mot en tant que nom qui exhibe un étant fixé. (Heidegger, 1976, 213)

" Objet » situé au-delà du reconnaissable " chose première » qui point entre les arbres, ce à quoi le sujet confère " nom et privilège au-dessus de l'ordinaire de sa banalité » est justement ce qu'aucun nom n'assigne définitivement à résidence dans la deuxième section du poème, ce dont la triple anaphore «The way...» ne dit l'absolue nouveauté qu'en signalant une sortie de la comparaison ontique, pointant l'impuissance de la figure à nommer ce qui apparaît, de sorte qu'elle se trouve réduite à décliner les modalités de l'apparaître sous l'aspect de l'apport lexical («adds»), de la création (« creates »), et de la révélation (« reveals »).

Dans « The Poems of our Climate », Stevens observait déjà :

The imperfect is our paradise.

Note that, in this bitterness, delight,

Since the imperfect is so hot in us,

Lies in flawed words and stubborn sounds (Stevens, 179)

51 C'est en reconnaissant la faille au creux des mots et l'entêtement du phonème comme trace de l'intention de signifier que Stevens pratique cette forme paradoxalement active de résignement que Heidegger observe chez George lorsqu'il écrit :

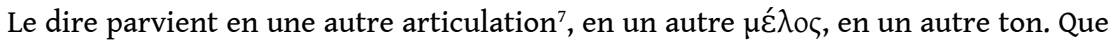
le résignement du poète soit expérimenté en ce sens, c'est ce qu'atteste le poème lui-même - qui dit le résignement cependant qu'il le chante. (Heidegger, 1976, 214) 


\section{BIBLIOGRAPHIE}

ADORNO, Theodor, Notes sur la littérature, traduit de l'allemand par Sibylle Muller, Paris, Flammarion, 1984.

AGAMBEN, Giorgio, Le Langage et la mort, traduit de l'italien par Marilène Raiola, Paris, Christian Bourgois, 1997.

CRITCHLEY, Simon, Things Merely Are: Philosophy in the Poetry of Wallace Stevens, London \& New York, Routledge, 2005.

DASTUR, Françoise, A la naissance des choses. Art, poésie et philosophie, La Versanne, Encre Marine, 2005.

DERRIDA, Jacques, Psyché, Invention de l'autre, Paris, Galilée, 1987-1998.

HEIDEGGER, Martin, Acheminement vers la parole, traduit de l'allemand par J. Beaufret, W. Brokmeier, F. Fédier, Paris, Gallimard, 1976.

---, Chemins qui ne mènent nulle part, traduit de l'allemand par W. Brokmeier, Paris, Gallimard, 1962.

---, Questions 1 et 2, traduit de l'allemand par K. Axelos, J. Beaufret et al., Paris, Gallimard, 1968.

KERMODE, Frank, dir., Wallace Stevens : Collected Poetry and Prose, New York, Literary Classics of the United States, 1997.

LACOUE-LABARTHE, Philippe, La Poésie comme expérience, Paris, Christian Bourgois, 1997.

PORTE, Joel, dir., Ralph Waldo Emerson : Essays and Lectures, New York, Literary Classics of the United States, 1983.

\section{NOTES}

1. Toutes les citations de Critchley sont traduites par mes soins.

2. Je traduis. Cette formule figure dans la conférence de Stevens intitulée «The Figure of the Youth as Virile Poet » (1943).

3. Notion développée par Stevens dans l'essai intitulé « The Noble Rider and the Sound of Words $\gg(1941)$.

4. Dans ce texte Adorno dénonce la lecture heideggerienne qui, en se concentrant exclusivement sur le signifié, évacue ce qui constitue le trait définitoire du texte de Hölderlin : sa poéticité.

5. Je m'efforcerai dans les derniers paragraphes de la présente étude d'examiner à la lecture de Stevens les prolongements de ce concept mis en œuvre par Lacoue-Labarthe dans La Poésie comme expérience.

6. Je me réfère aux vers suivants : «But suppose the disorder of truths should ever come/ To an order, most Plantagenet, most fixed ...»

7. Je modifie légèrement la traduction de Beaufret, Brokmeier et Fédier, qui rendent « Gliederung » par « membrure ». 


\section{RÉSUMÉS}

Il s'agit dans cet article de montrer que la « vision officieuse de l'être » que nous propose Wallace Stevens ne se résume pas à faire éprouver le sentiment du monde tel qu'il est en un parfait ajointement de l'imagination à la réalité. Après avoir exploré les liens entre l'ontologie heideggérienne et la poésie de Stevens "en temps de nécessité », ce travail interroge la fonction originante du poème à la lumière de la théorisation de la deixis et de la voix à laquelle se livre Agamben, et du rôle spécifique qu'y revêt la syllabe. A la lecture de Lacoue-Labarthe, il s'achève par un examen de la poésie de Stevens comme expérience concomitante au retrait de l'être.

This paper aims to show that Wallace Stevens' "unofficial view of being" does not simply convey a sense of the world as it is in the perfect coincidence of imagination and reality. After exploring the connection between Heideggerian ontology and Stevens' poetry "in times of need", this paper examines the originating function of Stevens' poems in light of Agamben's theorization of deixis and voice, and of the specific role assigned to the syllable. Following Lacoue-Labarthe, I conclude with a discussion of Stevens' poetry as experience concomittant with the withdrawal of being.

\section{INDEX}

Mots-clés : Agamben, deixis, épistémologie, expérience, Heidegger, Lacoue-Labarthe, ontologie, origine, syllabe, voix

Keywords : Agamben, deixis, epistemology, experience, Heidegger, Lacoue-Labarthe, ontology, origin, syllable, voice

\section{AUTEUR}

\section{AXEL NESME}

Université Lyon 2 